Este libro forma parte del acervo de la Biblioteca Jurídica Virtual del Instituto de Investigaciones Jurídicas de la UNAM

\title{
ACEPTAR EL RETO: LOS ENSAYOS DE INFEGGIÓN INTENCIONAL PARA LA VACUNA CONTRA EL COVID-19 PUEDEN JUSTIFICARSE ÉTICAMENTE ${ }^{1}$
}

\author{
Kyle Ferguson \\ Arthur GAPLAN
}

Es poco probable que la pandemia de COVID-19 termine hasta que haya una vacuna segura, eficaz y ampliamente distribuida. ¿Qué tan pronto pueden los investigadores lograr este objetivo? La respuesta depende en gran medida de las estrategias que los investigadores estén dispuestos a adoptar. Una estrategia potencial es realizar estudios de infección intencional en humanos, ${ }^{2}$ en los que los investigadores administran una vacuna experimental a voluntarios sanos y luego prueban (o "retan") la vacuna exponiendo deliberadamente a los voluntarios al virus. Aunque un número creciente de voces están pidiendo a los investigadores que empleen esta estrategia, la propuesta está generando un acalorado debate sobre la ética de dicha investigación.

En los ensayos de vacunas convencionales, los investigadores administran una vacuna experimental o un placebo a decenas de miles de voluntarios y luego esperan a que la naturaleza haga su trabajo, un proceso que normalmente lleva años. A medida que las curvas se aplanan, las condiciones para la investigación de vacunas convencionales empeoran, ${ }^{3}$ al reducir la tasa de infección natural y ralentizar el proceso de descubrir si la vacuna

\footnotetext{
1 Una versión anterior de este texto se publicó en el Hastings Bioethics Forum del Hastings Center. Se reproduce aquí con el permiso del Hastings Center y de los autores. Traducción de Gustavo Ortiz Millán.

2 Aunque en ocasiones se ha traducido human challenge studies al español como "estudios clínicos de provocación" o "estudios de reto", aquí se traduce como "estudios de infección intencional en humanos", que describe mejor en qué consisten los estudios o ensayos clínicos que involucran la exposición intencional a la enfermedad. (N. del t.).

3 Salzman, Sony, "Vaccine paradox: Will 'flattening the curve' stymie the chances of developing a coronavirus vaccine quickly?", ABC News, 2 de junio de 2020, disponible en: https://abcnews.go.com/Health/vaccine-paradox-flattening-curve-stymie-chances-developing-coronavirus/ story:? $\mathrm{id}=70885643$.
} 
Este libro forma parte del acervo de la Biblioteca Jurídica Virtual del Instituto de Investigaciones Jurídicas de la UNAM

funcionó. Moralmente, quienes realizan ensayos de vacunas deben instar a sus sujetos a evitar la infección, alargando así el tiempo necesario para completar el estudio. Los estudios de infección intencional toman mucho menos tiempo, requieren muchos menos voluntarios, no están atados a las tasas naturales de infección y, por lo tanto, pueden acelerar el progreso. Pero podrían poner a los voluntarios en mayor riesgo que los ensayos de vacunas convencionales.

Los defensores creen que hay formas de reducir los riesgos a niveles aceptables. Plotkin y Caplan proponen reclutar solo voluntarios jóvenes y sanos. ${ }^{4}$ Eyal, Lipsitch y Smith proponen además el reclutamiento en áreas con altas tasas de infección para minimizar el riesgo adicional para los voluntarios. ${ }^{5}$ Shah, Miller y sus colegas argumentan que los riesgos de infecciones controladas por SARS-GoV-2 son comparables a los riesgos aceptados regularmente en la investigación. ${ }^{6}$ Pero los opositores creen que los riesgos siguen siendo demasiado altos para que valga la pena correrlos.

En un reciente ensayo en el Hastings Bioethics Forum, Ruth Macklin se opone al uso de estudios de infección intencional en humanos en la investigación de la vacuna para el COVID-19. ${ }^{7}$ Como otros que se oponen a tales estudios, ${ }^{8}$ Macklin basa su caso en un principio fundamental de la ética de la investigación, que obliga moralmente a los investigadores a minimizar los riesgos para los voluntarios. En su opinión, exponer a los voluntarios al SARS-CoV-2 no sería ético ya que no existe un tratamiento aceptado para el COVID-19. Macklin escribe que "una advertencia de larga data en el uso de [estudios de infección intencional en humanos] es un requisito previo de que debe existir un tratamiento aceptado para la enfermedad en estudio. Ese requisito no se cumple actualmente en el caso de COVID-19". Aunque

4 Plotkin, Stanley A., y Caplan, Arthur, "Extraordinary diseases require extraordinary solutions", Vaccine, vol. 38, núm. 24, 19 de mayo de 2020, pp. 3987 y 3988, disponible en: https://doi.org/10.1016/j.vaccine.2020.04.039.

5 Eyal, Nir et al., "Human challenge studies to accelerate coronavirus vaccine licensure", The Fournal of Infectious Diseases, vol. 221, núm. 11, 1o. de junio de 2020, pp. 1752-1756, disponible en: https://doi.org/10.1093/infdis/jiaa152. Publicado el 31 de marzo de 2020.

6 Shah, Seema et al. "Ethics of controlled human infection to address COVID-19", Science, vol. 368, núm. 6493, pp. 832-834, 22 de mayo de 2020, DOI: 10.1126/science.abc1076.

7 Macklin, Ruth, "Human challenge studies for COVID-19 vaccine: Questions about benefits and risks", Hastings Bioethics Forum, 15 de junio de 2020, disponible en: https:// wwre: thehastingscenter.org/human-challenge-studies-for-covid-19-vaccine-questions-about-benefits-andrisks/. (La versión extendida de este ensayo se publica en español en este mismo volumen).

8 Dawson, Liza et al., "Severe acute respiratory syndrome coronavirus 2 human challenge trials: too risky, too soon", The Fournal of Infectious Diseases, vol. 222, núm. 3, 1o. de agosto de 2020, pp. 514-516, disponible en: https://doi.org/10.1093/infdis/jiaa314. 
Este libro forma parte del acervo de la Biblioteca Jurídica Virtual del Instituto de Investigaciones Jurídicas de la UNAM

Macklin plantea preocupaciones adicionales sobre el consentimiento informado y la selección justa de sujetos, ninguno de los cuales es exclusivo de los estudios de infección intencional, su principal preocupación es el riesgo. Ella concluye que "la prisa por comenzar los ensayos de vacunas de desafío humano para una enfermedad grave que carece de un tratamiento eficaz es éticamente injustificable".

No estamos de acuerdo. Sin duda, respaldamos el principio de minimizar los riesgos en la investigación. Sin embargo, creemos que los estudios de infección intencional del SARS-CoV-2 pueden cumplir con el requisito de minimizar los riesgos. Debido a esto, y debido a los enormes beneficios sociales que prometen, los ensayos de vacunas de infección intencional en humanos para COVID-19 pueden estar éticamente justificados.

El argumento de Macklin pone mucho peso en el hecho de que no existe un tratamiento aceptado para COVID-19. Sin embargo, distingamos dos cuestiones: por un lado, si existe un tratamiento; por otro, si se minimizan los riesgos. Estas dos no son la misma cosa. Por lo tanto, la "advertencia de larga data" en los estudios de infección intencional de que exista un tratamiento aceptado es simplemente un sustituto del requisito de minimizar los riesgos, no un requisito en sí mismo. Hay otras formas de minimizar los riesgos. Ofrecemos cinco razones para pensar que los estudios de infección intencional del SARS-CoV-2 pueden minimizar los riesgos y satisfacer las demandas de la ética de la investigación.

Primero, los estudios de desafío han recibido la aprobación de comités de ética de la investigación [institutional review boards], incluso sin un tratamiento aceptado para la enfermedad en cuestión. Por ejemplo, han tenido un papel importante en el establecimiento de candidatos a vacunas para el dengue. ${ }^{9}$ En un estudio publicado en 2016, los investigadores infectaron a voluntarios con una cepa del virus del dengue para probar la vacuna experimental TV003. En ese estudio, la vacuna protegió a todos los receptores del dengue, lo que significaba que se justificaban estudios adicionales. ${ }^{10}$ Este fue un avance positivo después de que otra vacuna, Dengvaxia, causara gran daño y controversia en Filipinas. ${ }^{11}$ Independientemente del futuro de

9 Kirkpatrick, Beth D. et al., "The live attenuated dengue vaccine TV003 elicits complete protection against dengue in a human challenge model", Science Translational Medicine, vol. 8, núm. 330, pp. 330ra36, 16 de marzo de 2016, DOI: 10.1126/scitranslmed.aaf1517.

10 Kallas, Esper G. et al., "Safety and immunogenicity of the tetravalent, live-attenuated dengue vaccine Butantan-DV in adults in Brazil: a two-step, double-blind, randomised placebo-controlled phase 2 trial", The Lancet Infectious Diseases, vol. 20, núm. 7, pp. 839-850, 2020, DOI: https://doi.org/10.1016/S1473-3099(20)30023-2.

11 Chatterjee, Patralekha, "Dengvaxia researcher charged", The Lancet Infectious Diseases, vol. 19, núm. 6, pp. 584 y 585, 2019, DOI: https://doi.org/10.1016/S1473-3099(19)30212-9. 
Este libro forma parte del acervo de la Biblioteca Jurídica Virtual del Instituto de Investigaciones Jurídicas de la UNAM

TV003 (la vacuna aún se está estudiando), el punto es que los investigadores realizaron estudios éticos de infección intencional del dengue a pesar de que no había, y todavía no hay, un tratamiento médico específico para la enfermedad.

En segundo lugar, los riesgos a los que se enfrentan los voluntarios dependen del agente de infección [challenge agents] que utilicen los investigadores. Un agente de infección es una versión específica de un patógeno seleccionado o diseñado y fabricado ${ }^{12}$ para su uso en estudios de infección intencional. El objetivo habitual es producir una infección, no una enfermedad ni daños en el cuerpo. En el estudio de infección intencional del dengue, los investigadores utilizaron un virus de infección, rDEN2D30, que derivaron de una cepa particular aislada durante un brote notable por sus casos leves. En los estudios de infección intencional para la malaria, ${ }^{13}$ los investigadores prueban las cepas de la malaria antes de infectar a los voluntarios para asegurarse de que están usando cepas que respondan a los tratamientos. Nadie puede decir con certeza que los estudios de infección intencional con SARS-CoV-2 son demasiado riesgosos sin saber más sobre el agente de infección y las dosis. En el contexto de COVID-19, el doctor Anthony Fauci le dijo recientemente a Politico: "Estamos haciendo dosis de infección" ${ }^{14}$ Es natural, pero es un error, pensar que los riesgos serían necesariamente los asociados con los peores casos de COVID-19.

En tercer lugar, los tratamientos aceptados, como la mayoría de las herramientas con las que vivimos, tienen tasas de fracaso distintas de cero. ${ }^{15}$ Por lo tanto, incluso cuando existen tratamientos, siempre queda cierto riesgo en los estudios de infección intencional. Minimizar los riesgos no significa eliminarlos.

Cuarto, otros tipos de investigación de vacunas se aprueban regularmente a pesar de los graves riesgos. Por ejemplo, los voluntarios en los en-

12 Catchpole, Andrew P. et al., "The manufacturing of human viral challenge agents for use in clinical studies to accelerate the drug development process", BMC Research Notes, vol. 11, núm. 620 (2018).

13 Matuschewski, K. y Borrmann, S., 2019, "Controlled Human Malaria Infection (CHMI) Studies: Over 100 Years of Experience with Parasite Injections", en Ariey, F. et al. (comps.), Malaria Control and Elimination, Methods in Molecular Biology, vol. 2013, Nueva York, Humana, pp. 91-101, disponible en: https://doi.org/10.1007/978-1-4939-9550-9_7.

14 Owermohle, Sarah, 2020, "White House pressure for a vaccine raises risk the U. S. will approve one that doesn't work", Politico, 15 de junio de 2020, disponible en: https://wrere. politico.com/newes/2020/06/15/pressure-coronavirus-vaccine-risk-approval-316094.

15 Lowe, Derek, "The latest on drug failure and approval rates", Science Translational Medicine, 9 de mayo de 2019, disponible en: https://blogs.sciencemag.org/pipeline/archives/2019 105/09/the-latest-on-drug-failure-and-approval-rates. 
Este libro forma parte del acervo de la Biblioteca Jurídica Virtual del Instituto de Investigaciones Jurídicas de la UNAM

sayos de vacunas convencionales enfrentan riesgos de sucesos adversos ${ }^{16}$ de los que ningún agente de rescate puede salvarlos. Estos riesgos son minimizados y habitualmente aceptados por investigadores, voluntarios y comités de ética de la investigación. ¿Por qué, entonces, la falta de un tratamiento aceptado debería ser un factor decisivo para los estudios de infección intencional? Sospechamos que actúan rarezas moral-psicológicas, no principios. No hay ninguna razón convincente para percibir el riesgo de daño derivado de una infección deliberada como moralmente peor que el riesgo de daño derivado de una vacuna experimental.

Finalmente, durante el primer pico de la pandemia, todos no solo permitimos, sino que elogiamos ${ }^{17}$ a los médicos, enfermeras, técnicos de emergencias sanitarias, personal de limpieza y otros que asumieron grandes riesgos para brindar atención a los pacientes con COVID-19. Estos riesgos eran mucho mayores que los que enfrentarían los voluntarios del estudio, pero nuestras comunidades los juzgaron como dignos de tomarse dado todo lo que está en juego. No estamos sugiriendo que el límite superior de riesgo aceptable en la investigación sea o deba ser el mismo que en otros caminos de la vida. Más bien, citamos este heroísmo como un recordatorio del valor moral de la toma de riesgos. El altruismo y el espíritu de servicio público parecen animar a los voluntarios de los estudios de infección intencional. ${ }^{18}$ Aquellos que se oponen a los estudios de infección intencional del SARSCoV-2 no solo calculan mal los riesgos; también juzgan mal el valor que impulsa la participación.

16 Herper, Matthew, "He experienced a severe reaction to Moderna's COVID-19 vaccine candidate. He's still a believer", Stat, 26 de mayo de 2020, disponible en: https://wrere. statnews.com/2020/05/26/moderna-vaccine-candidate-trial-participant-severe-reaction/.

17 "The heroism of health workers in the coronavirus crisis", The New Fork Times, 26 de marzo de 2020, disponible en: https://wrwenytimes.com/2020/03/26/opinion/letters/coronavirus-health-care.html.

18 Morrison, Josh, "Challenge trials can speed development of a COVID-19 vaccine. Planning for them needs to start now", Stat, 28 de mayo de 2020, disponible en: https://wrere. statnews.com/2020/05/28/challenge-trials-speed-development-covid-19-vaccine-start-planning-now/. 